\title{
MicroRNA-144 suppresses tumorigenesis of hepatocellular carcinoma by targeting AKT3
}

\author{
YING MA*, XING-GUO SHE*, YING-ZI MING, QI-QUAN WAN and QI-FA YE \\ Department of Transplant Surgery, The Third Affiliated Hospital, Central South University, \\ Changsha, Hunan 410013, P.R. China
}

Received February 12, 2014; Accepted October 3, 2014

DOI: $10.3892 / \mathrm{mmr} .2014 .2844$

\begin{abstract}
Aberrant expression of microRNAs (miRNAs) has been shown to be associated with the progression and metastasis of cancer. Dysregulation of miR-144 has been observed in numerous types of cancer; however, the exact role of miR-144 in hepatocellular carcinoma (HCC) remains unclear. The present study observed that miR-144 was downregulated in HCC tissues and cell lines. Forced overexpression of miR-144 suppressed proliferation, migration and invasion of HCC cells. AKT3 was identified as a direct target of miR-144 in HCC, and this was confirmed by a luciferase activity assay and western blot analysis. Overexpression of AKT3 in miR-144 transfected HCC cells effectively reversed the tumor suppressive effects of miR-144. Furthermore, AKT3 expression levels were inversely correlated with miR-144 expression levels in HCC tissues. In conclusion, the results of the present study suggest that miR-144 may act as a tumor suppressor in HCC by targeting AKT3, and miR-144 may be a potential therapeutic candidate for HCC.
\end{abstract}

\section{Introduction}

Hepatocellular carcinoma (HCC) is the third most common cause of cancer-associated mortality, and affects $>500,000$ individuals annually, worldwide (1). Hepatocarcinogenesis is a complicated, multi-step process that is associated with various genetic and epigenetic alterations $(2,3)$. Despite recent advances in functional genomics, the molecular pathogenesis of HCC remains to be elucidated. Therefore, identifying novel molecules associated with the development of HCC is required in order to combat this aggressive malignancy.

MicroRNAs (miRNAs) are an abundant family of small endogenous non-coding RNAs, with essential roles in the

Correspondence to: Mr. Qi-Fa Ye, Department of Transplant Surgery, The Third Affiliated Hospital, Central South University, 138 Tongzipo Road, Changsha, Hunan 410013, P.R. China

E-mail: yeqifa_hn@163.com

${ }^{*}$ Contributed equally

Key words: hepatocellular carcinoma, microRNA-144, AKT3, growth, invasion regulation of gene expression. miRNAs bind to the 3'-untranslated region (3'-UTR) of genes by imperfect base-pairing to complementary sequences, and induce target mRNA degradation or translational repression (4). Emerging evidence has demonstrated the aberrant expression of miRNAs in numerous types of cancer, including HCC (5-7). Among them, miR-144 is aberrantly expressed in various malignancies, including bladder (8), colorectal (CRC) (9) and lung cancer (10), as well as HCC (11). There are various signaling pathways through which miR-144 plays a critical role in the development of numerous cancers, by targeting different molecules involved in those signalling pathways.

A number of these target molecules have been experimentally identified, including enhancer of zeste homolog 2 (EZH2), mammalian target of rapamycin (mTOR) and Zinc finger X-chromosomal protein (8-10).

In the present study, the effects of miR-144 on the proliferation, migration and invasion of HCC cells were investigated, and its downstream target was identified.

\section{Materials and methods}

Patient samples. A total of $29 \mathrm{HCC}$ patients were enrolled in the present study from the Third Affiliated Hospital (Changsha, China). The present study was approved by the Ethics Committee of the Third Affiliated Hospital, and informed consent was provided by each patient. The samples were obtained during surgery, without pre-surgical chemotherapy or radiation therapy. The tissues were immediately snapfrozen and stored at $-80^{\circ} \mathrm{C}$

Cell culture and transfection. The HepG2, HuH7 and SMMC-7721 human HCC cell lines, and the HL-7702 normal human hepatocyte cell line were obtained from the Shanghai Institute of Cell Biology (Shanghai, China). The cells were cultured in Dulbecco's modified Eagle's medium (Invitrogen Life Technologies, Carlsbad, CA, USA) and were incubated at $37^{\circ} \mathrm{C}$ in an atmosphere containing $5 \% \mathrm{CO}_{2}$. Transfections of the overexpression plasmids and the corresponding control were performed using Lipofectamine 2000 (Invitrogen Life Technologies), according to the manufacturer's instructions.

RNA extraction and quantitative polymerase chain reaction (qPCR). SYBR Green (Takara Bio, Inc., Otsu, Japan) 
reverse transcription (RT)-qPCR (using RT kit; Toyobo Co., Ltd., Osaka, Japan) was performed using an ABI StepOne machine (Applied Biosystems Life Technologies, Foster City, CA, USA). Fold change was calculated using the $2^{-\Delta \Delta \mathrm{Ct}}$ method. The primer sequences used for RT-qPCR were as follows: AKT3 sense 5'-AAAACAGAACGACCAAAG-3' and antisense 5'-TCTGCTACAGCCTGGATA-3'; and GAPDH sense 5'-CCACTCCTCCACCTTTGAC-3' and antisense 5'-ACCCTGTTGCTGTAGCCA-3'. The primers for miR-144 (cat.no HmiRQP0190) and the control U6 (cat. no HmiRQP9001) were obtained from GeneCopoeia, Inc. (Rockville, MD, USA). Tissue samples were homogenized, and miRNA was extracted using an All-In-One microRNA Extraction kit (GeneCopoeia, Inc.). RNA was extracted using TRIzol (Invitrogen Life Technologies). The expression levels were normalized to GAPDH or U6. Reactions were incubated for $10 \mathrm{~min}$ at $95^{\circ} \mathrm{C}$, followed by 40 cycles of $95^{\circ} \mathrm{C}$ for $10 \mathrm{sec}$; $60^{\circ} \mathrm{C}$ for $20 \mathrm{sec}$ and $72^{\circ} \mathrm{C}$ for $10 \mathrm{sec}$. The temperature was then increased from 68 to $95^{\circ} \mathrm{C}$ to produce a melting curve.

Plasmid construction and luciferase activity assay. miR-144 (pre-miR-144) and pre-miR negative control (NC) were purchased from Ambion Life Technologies (Carlsbad,CA,USA). pcDNA3-AKT3 was generated using the following primers: Sense: 5'-GGGGTACCCAAACCCTAAAGCTGATA-3' and antisense: 5'-CCCTCGAGACAGTAGCAGCAACAGCA-3'. The PCR product was inserted into pcDNA3.0 within the KpnI and $X h o I$ restriction sites (Invitrogen Life Technologies). The 3'-UTR of AKT3 mRNA was amplified using the following primers: Sense: 5'-CCCTCGAGTCCCTCAGTGAAGGCTAA-3' and antisense: 5'-TTGCGGCCGCTCTTCAGCCATCAGAGGT-3'. The PCR product and its mutant were inserted into the dual luciferase reporter vector psiCHECK2 (Promega Corporation, Madison, Wi, USA), within the XhoI and Not I restriction sites (Promega Corporation).

The SMMC-7721 cells were co-transfected with the luciferase reporter vector, containing the 3'-UTR of AKT3 [wild type (WT) or mutant (Mut)], and pre-miR-144 or pre-miR NC. The luciferase activities were determined $48 \mathrm{~h}$ post-transfection using the Dual-Luciferase Reporter Assay system (Promega Corporation).

Analysis of cell viability and colony formation. The cell viability was examined using a Cell Counting kit-8 (CCK-8; Beyotime, Shanghai, China), according to the manufacturer's instructions, following transfection with pre-miR-144 or pre-miR NC at the indicated time points. The absorbance was measured at $450 \mathrm{~nm}$ by the absorbance reader (Thermo Fisher Scientific, Waltham, MA, USA). For colony formation analysis, 500 transfected SMMC-7721 cells were seeded in each well of 6-well plates. The surviving colonies were stained with $0.5 \%$ crystal violet and counted, following a two week incubation. The experiments were performed in triplicate. For the observation of cells a CK12 inverted microscope was used (Olympus Corporation, Tokyo, Japan) according to the manufacturer's instructions.

Analysis of cell invasion and migration. Cell invasion and migration were examined by a Transwell assay (EMD
Millipore, Billerica, MA, USA). Filters in the upper chamber were pre-coated with Matrigel (BD Biosciences, San Jose, CA, USA) for the invasion assays. A total of $5 \times 10^{4}$ cells were seeded in the upper chamber, and the lower chamber was filled with culture media supplemented with $10 \%$ fetal bovine serum (Invitrogen, Foster City, CA, USA) . The invaded and migrated cells on the bottom surface were stained with $0.5 \%$ crystal violet and counted, at the indicated time points. The experiments were performed in triplicate, and four fields were counted per filter.

Western blot analysis. The antibodies used were as follows: Rabbit anti-human polyclonal AKT3 antibody and Rabbit antihuman monoclonal GAPDH antibody (all 1:1,000; from Cell Signaling Technology, Inc., Beverly, MA, USA). The protein concentrations were determined by using BCA protein assay kit (Beyotime). The cell lysates were prepared using a lysis buffer (pH 7.5, $50 \mathrm{mM}$ Tris-HCl, $5 \mathrm{mM}$ EDTA, 0.5\% NP-40 and $150 \mathrm{mM} \mathrm{NaCl}$ ). The protein samples were separated by $10 \%$ SDS-PAGE and transferred to polyvinylidene fluoride membranes (Millipore, MA, USA). The membranes were then probed with primary antibodies, followed by an incubation with the appropriate horseradish peroxidase-labeled secondary antibodies. The blots were visualized using an Enhanced Chemiluminescence reagent (GE Healthcare Life Sciences, Chalfont, UK). The protein expression levels were normalized to GAPDH and quantified by optical densitometry using Image J Software 1.46 (National Institute of Health, Bethesda, MA, USA).

Statistical analysis. The data are presented as the mean \pm standard deviation. A one-way analysis of variance or Student's $t$-test was performed to determine statistical significance. SPSS software, version 15.0 (SPSS Inc., Chicago, IL, USA) was used for the statistical analyses. $\mathrm{P}<0.05$ was considered to indicate a statistically significant difference. Spearman's correlation analysis was used to analyze the correlation of miR-144 and AKT3.

\section{Results}

miR-144 is downregulated in HCC tissues and cell lines. A qPCR analysis of miR-144 was conducted using paired HCC and adjacent normal tissues from 29 HCC patients. miR-144 was significantly downregulated in the HCC tissues, as compared with the normal tissues (Fig. 1A). Furthermore, the expression levels of miR-144 in the three human HCC cell lines: HepG2, HuH7 and SMMC-7721, and the HL-7702 normal human hepatocyte cell line, were examined by qPCR. Concordant with the tissue results, miR-144 was markedly downregulated in the HCC cell lines, as compared with the normal hepatocytes (Fig. 1B).

Suppression of HCC cell proliferation by $m i R-144$. To explore the function of miR-144 in tumor proliferation, the SMMC-7721 cells were transfected with miR-144 or a control miRNA. miR-144 overexpression significantly suppressed the proliferation of SMMC-7721 cells, as determined by a CCK-8 assay (Fig. 2A). The cells transfected with miR-144 formed fewer colonies, as compared with the miR-NC group (Fig. 2B). The effects of miR-144 overexpression were validated by qPCR (Fig. 2C). 

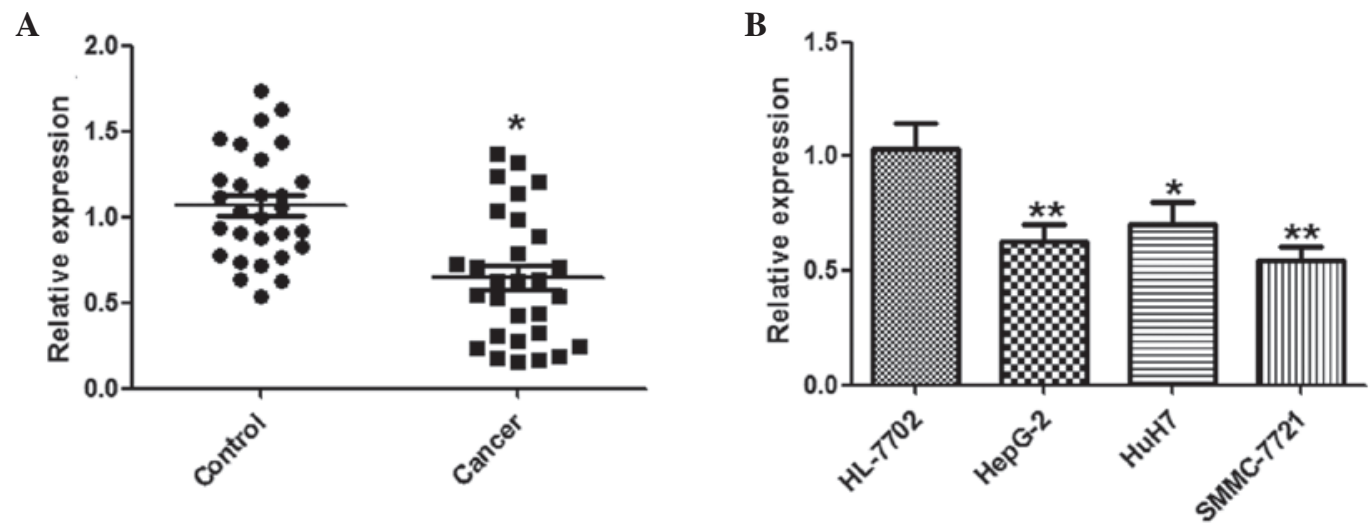

Figure 1. miR-144 was downregulated in HCC tissues and cell lines. (A) miR-144 expression levels were determined by qPCR in the tumor and adjacent non-cancerous tissues. (B) miR-144 expression levels were determined by qPCR in three human HCC cell lines: HepG-2, HuH7 and SMMC-7721, and HL-7702 the normal human hepatocyte cell line. ${ }^{*} \mathrm{P}<0.05$ and ${ }^{* *} \mathrm{P}<0.01$, compared with the controls. miR, microRNA; HCC, hepatocellular carcinoma; qPCR, quantitative polymerase chain reaction.
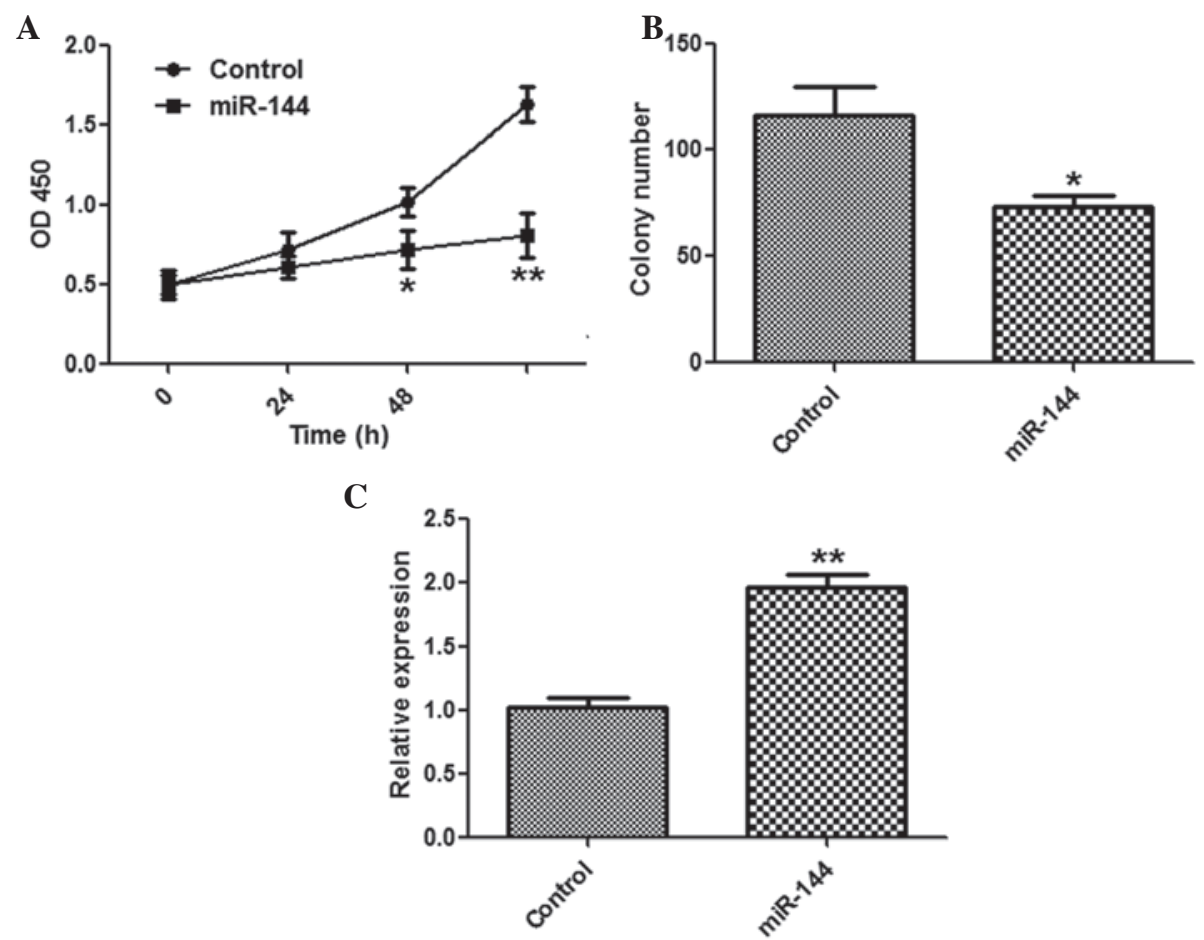

Figure 2. Suppression of HCC cell proliferation by miR-144. (A) miR-144 reduced the viability of the SMMC-7721 HCC cells, as assessed by a Cell Counting kit-8 assay. (B) Colony formation assay of the SMMC-7721 cells. (C) Effects of miR-144 overexpression were validated by quantitative polymerase chain reaction. Theses data were drawn from three independent experiments. ${ }^{*} \mathrm{P}<0.05$ and ${ }^{* *} \mathrm{P}<0.01$, compared with the controls. HCC, hepatocellular carcinoma; miR, microRNA; OD, optical density.

Inhibitory effects of miR-144 on invasion and migration of HCC cells. To determine whether miR-144 has a crucial role in cell invasion and migration, in vitro assays were performed. The number of invasive and migratory cells were significantly reduced in the group overexpressing miR-144, as compared with the group transfected with control miRNA (Fig. 3A and B).

AKT3 is a target of miR-144 in HCC cells. TargetScan was used to search for potential targets of miR-144, and AKT3 was identified as a potential target (Fig. 4A). miR-144 overexpression significantly inhibited the luciferase activity of the AKT3 WT 3'-UTR, but not the AKT3 Mut 3'-UTR (Fig. 4B). In addition, overexpression of miR-144 significantly suppressed the mRNA and protein expression levels of AKT3 (Fig. 4C and D).

AKT3 overexpression attenuates the suppressive effects of $m i R-144$. It was further investigated whether overexpression of AKT3 may reverse the suppressive effects of miR-144. A CCK8 assay (Fig. 5A), and invasion and migration assays (Fig. 5B and $\mathrm{C}$ ) showed that overexpression of AKT3 markedly reversed the suppressive effects of miR-144 on HCC cells. The effects of pcDNA3-AKT3 were confirmed by qPCR (Fig. 5D).

AKT3 expression levels are inversely correlated with miR-144 in HCC tissues. Expression levels of AKT3 in the HCC and 
A

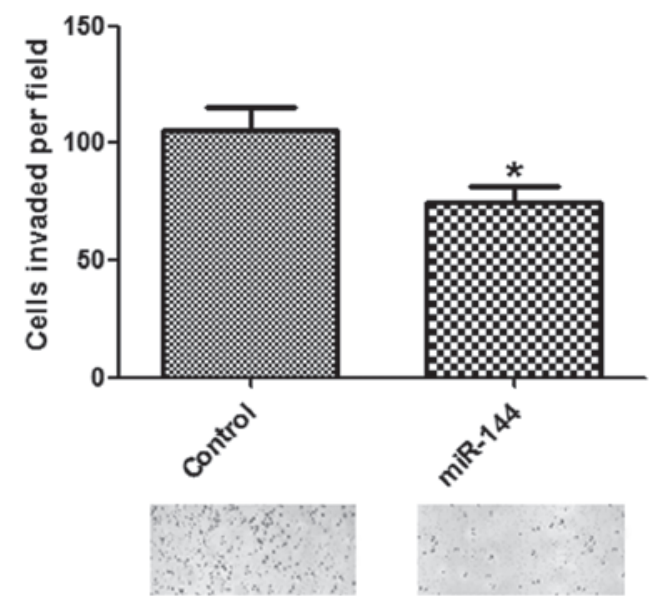

B

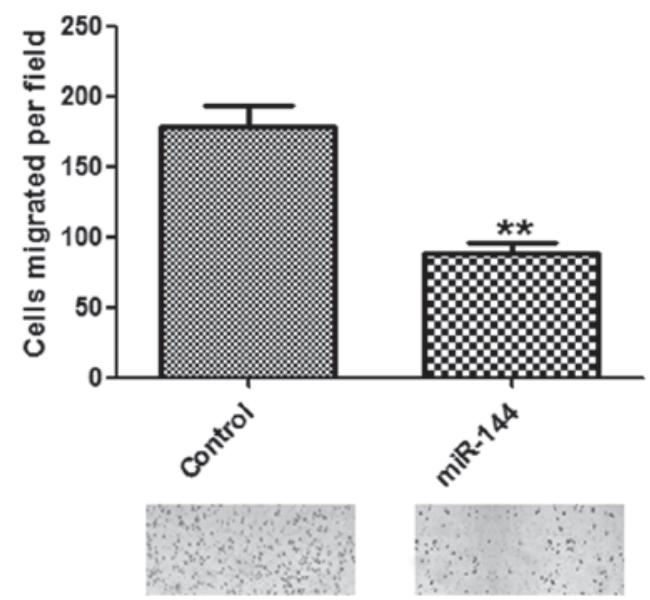

Figure 3. Inhibitory effects of miR-144 on the invasion and migration of HCC cells. (A) and (B) Overexpression of miR-144 was associated with reduced invasive and migratory abilities of the SMMC-7721 cells at various time points. These data were drawn from three independent experiments. ${ }^{*} \mathrm{P}<0.05$ and ${ }^{* *} \mathrm{P}<0.01$, compared with the controls. miR, microRNA; HCC, hepatocellular carcinoma.

A

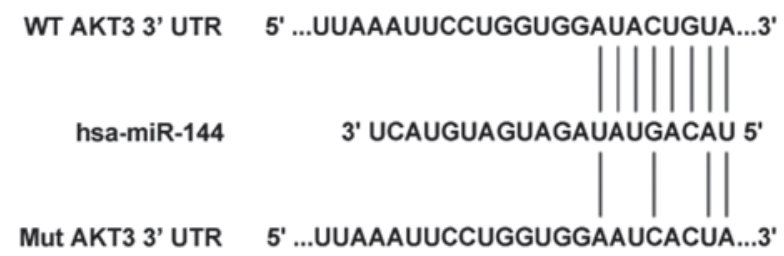

B
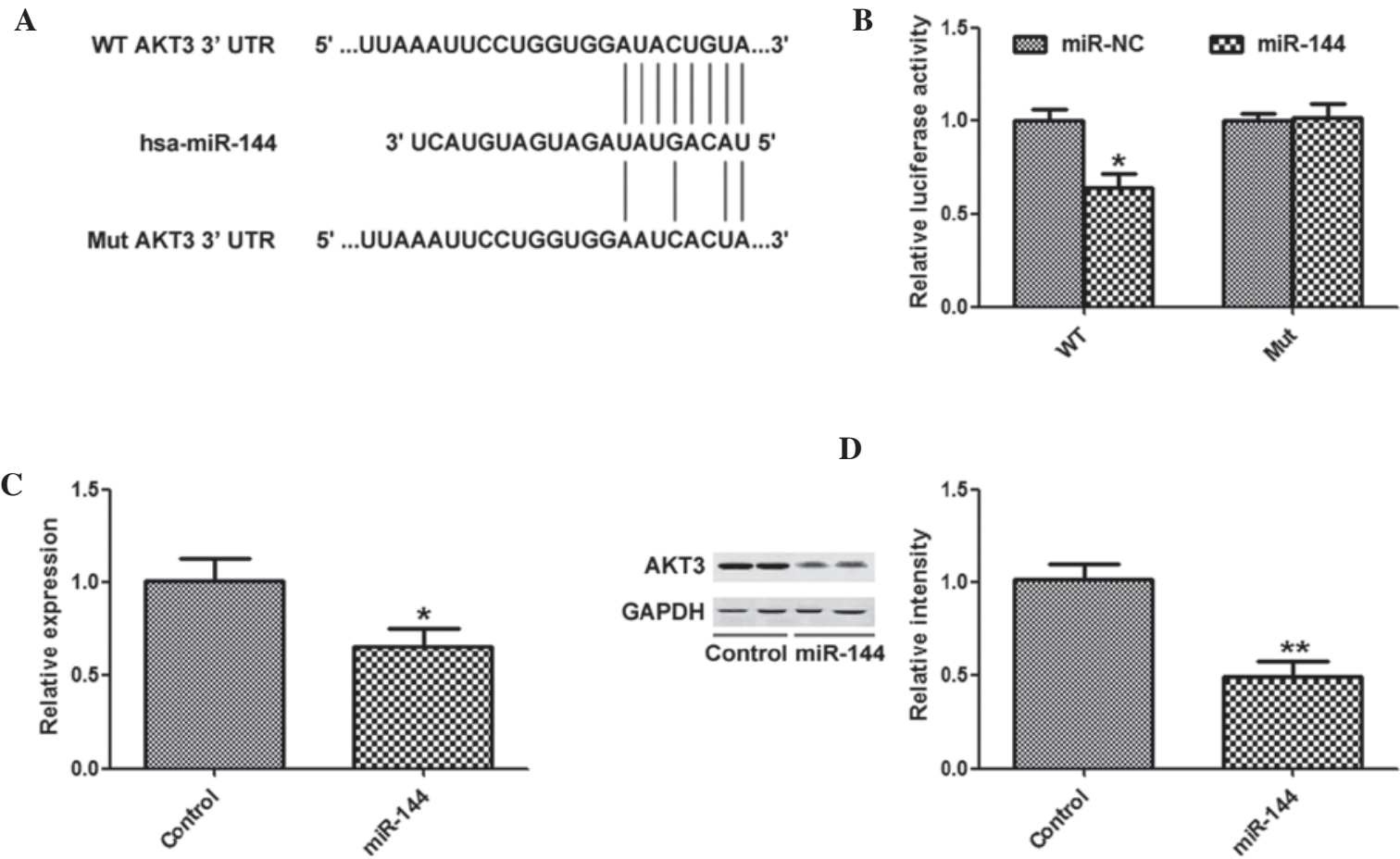

D

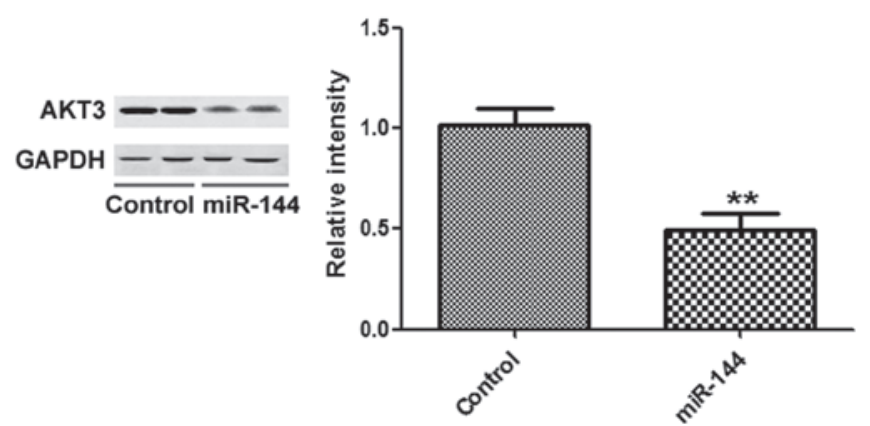

Figure 4. AKT3 was identified as a target of miR-144 in hepatocellular carcinoma cells. (A) Schematic construction of WT and Mut miR-144 binding sequences in the 3'-UTR of AKT3. (B) Suppressed luciferase activity of WT 3'-UTR of AKT3 by miR-144 overexpression. (C) Expression levels of AKT3 mRNA were detected by quantitative polymerase chain reaction of the SMMC-7721 cells transfected with miR-144 or control. (D) Protein expression levels were detected by western blot analysis of the SMMC-7721 cells transfected with miR-144 or control. ${ }^{*} \mathrm{P}<0.05$ and ${ }^{* *} \mathrm{P}<0.01$, compared with the control group. miR, microRNA; WT, wild type; Mut, mutant; 3'UTR, 3' untranslated region.

matched normal tissues were detected by qPCR. AKT3 expression levels were significantly increased in the HCC tissues, as compared with the matched normal tissues (Fig. 6A). Furthermore, AKT3 was negatively correlated with miR-144 expression levels in the same HCC tissues (Fig. 6B).

\section{Discussion}

The aim of the present study was to investigate the role of miR-144 in hepatocarcinogenesis, and to determine the underlying molecular mechanisms by which miR-144 exerts its function. miR-144 was shown to be downregulated in the HCC tissues and cell lines. Forced overexpression of miR-144 suppressed HCC cell proliferation, colony formation, and the invasive and migratory abilities of the cells. Theoretical targets of miR-144 were searched for using the target prediction software TargetScan. AKT3 was identified as a potential target and was further tested. Overexpression of AKT3 could partially attenuate the tumor suppressive effects of miR-144 in HCC cells. Furthermore, AKT3 was shown to be negatively 
A

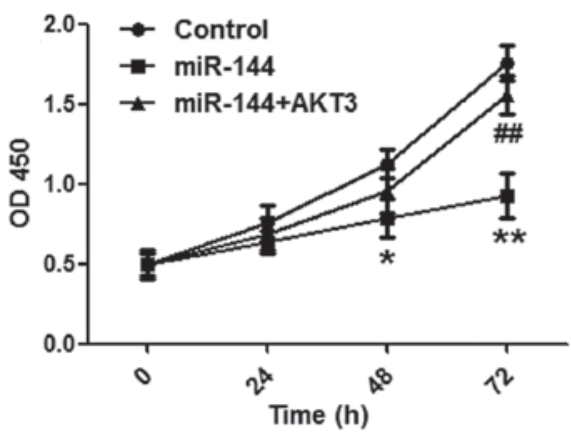

C

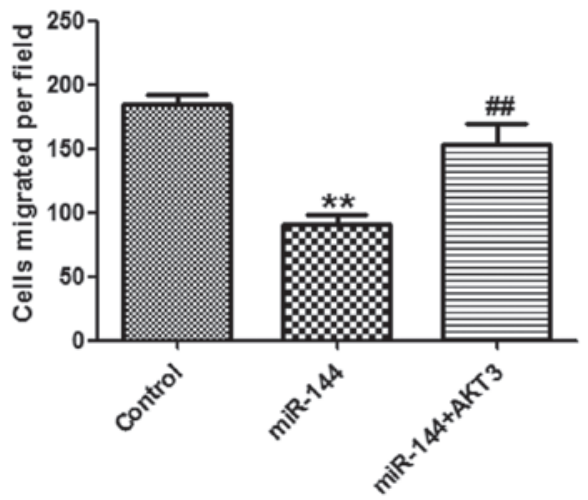

B

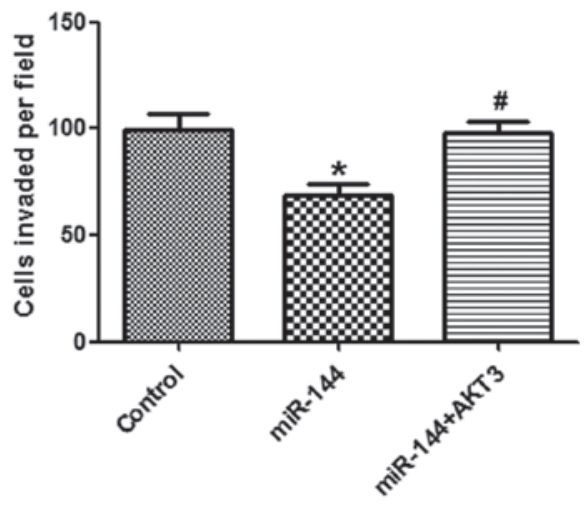

D

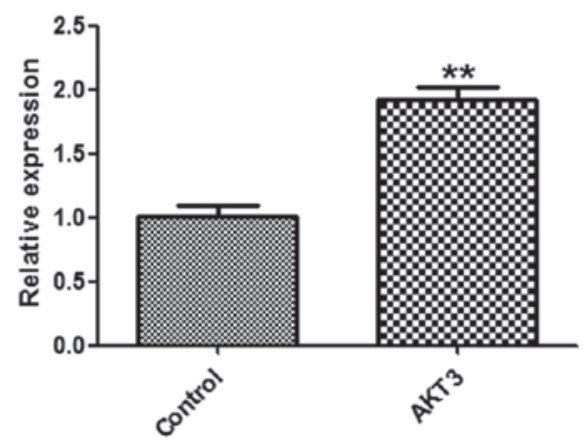

Figure 5. AKT3 overexpression attenuated the suppressive effects of miR-144. (A) SMMC-7721 hepatocellular carcinoma cells were co-transfected with miR-144 and AKT3, or transfected with miR-144 or the control vector. A Cell Counting kit-8 assay was used to detect proliferation. (B) Invasion and (C) migration assays of the SMMC-7721 cells co-transfected with miR-144 and AKT3, or transfected with miR-144 or the control vector. (D) Expression levels of AKT3 were detected by quantitative polymerase chain reaction in the SMMC-7721 cells transfected with AKT3, or the control. "P<0.05 and ${ }^{* *} \mathrm{P}<0.01$, compared with the control group. ${ }^{\#} \mathrm{P}<0.05$ and ${ }^{\# \#} \mathrm{P}<0.01$, compared with the miR-144 group. miR, microRNA; OD, optical density.

A

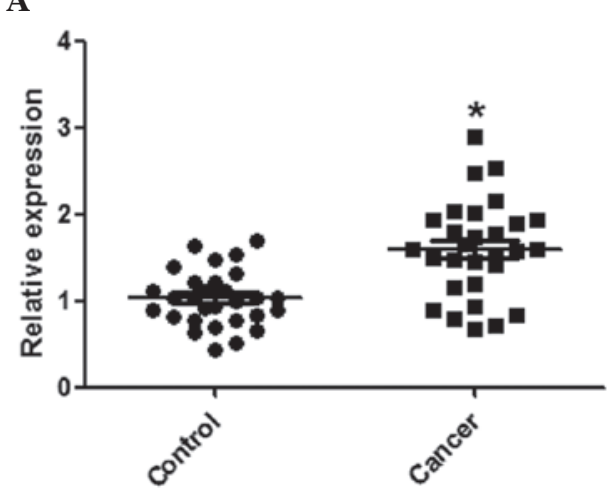

B



Figure 6. AKT3 expression levels were inversely correlated with miR-144 in HCC tissues. (A) The expression levels of AKT3 in the HCC and matched normal tissues were detected by quantitative polymerase chain reaction. (B) Correlation analysis between AKT3 mRNA expression levels and miR-144 expression levels in the HCC tissues (Spearman's correlation analysis, $\mathrm{r}=-0.469 ; \mathrm{P}<0.05$ ). ${ }^{*} \mathrm{P}<0.05$, compared with the controls. miR, microRNA; HCC, hepatocellular carcinoma.

correlated with miR-144 expression levels in the HCC tissues. These results suggest that miR-144 may have a potential role in the diagnosis and treatment of HCC.

The gene encoding miR-144 is located on chromosome 11 (9). Abnormal expression of miR-144 has previously been detected in nasopharyngeal carcinoma (NPC), CRC and HCC (9,11,12). In NPC, miR-144 was shown to be elevated and act as an oncogene. Repression of miR-144 substantially decreased cell proliferation, colony formation, invasion, migration, and tumor formation in nude mice. Furthermore, restoring miR-144 in miR-144-attenuated NPC cells exerted a strong tumorigenic role (12). Conversely, in CRC, miR-144 has been shown to be downregulated and miR-144 downregulation is associated with poor CRC prognosis. Suppression of miR-144 facilitated proliferation and increased rapamycin sensitivity of CRC cells, through activation of the mTOR signaling pathway (9). Furthermore, in bladder cancer, miR-144 served as a tumor suppressor by targeting EZH2 and regulating Wnt signaling (8). The present study expanded the role 
of miR-144 in HCC, and demonstrated that miR-144 acts as a tumor suppressor in HCC.

The AKT family includes three isoforms: AKT1, AKT2 and AKT3, which share a highly conserved domain structure and have similar roles in the regulation of cell proliferation, survival, metabolism and numerous other cellular functions (13). The function of AKT isoforms in mediating cancer development and progression is known to be orchestrated in a tissue-specific manner (14). Nassirpour et al (15) reported that hepatitis B virus-transformed cells had enhanced expression of AKT3, and specific downregulation of AKT3 was able to suppress migration and induce programmed cell death. They further demonstrated that AKT3, but not AKT1 or AKT2, was required and sufficient to promote migration and metastasis in certain HCCs. In concordance with these findings, the present study demonstrated that supplementation with AKT3 markedly attenuated the tumor suppressive effects of miR-144 on the proliferation, invasion and migration of HCC cells. Restoration of miR-144 may induce antitumor activities by targeting AKT3, thus suggesting that miR-144 could act as a tumor suppressor in HCC, which harbors decreased miR-144 expression levels.

In conclusion, the results of the present study showed that miR-144 bound to the 3'-UTR of AKT3, and overexpression of miR-144 in HCC cells decreased AKT3 mRNA and protein expression levels, resulting in the inhibition of cell proliferation, invasion and migration. Ectopic overexpression of AKT3 was able to attenuate the tumor suppressive characteristics induced by miR-144 overexpression, indicating that the regulation of tumorigenesis by miR-144 was mediated by targeting AKT3 in HCC.

\section{References}

1. Forner A, Llovet JM and Bruix J: Hepatocellular carcinoma. Lancet 379: 1245-1255, 2012.
2. Kong D, Chen $\mathrm{H}$, Chen $\mathrm{W}$, et al: Gene expression profiling analysis of hepatocellular carcinoma. Eur J Med Res 18: 44, 2013.

3. Aravalli RN, Steer CJ and Cressman EN: Molecular mechanisms of hepatocellular carcinoma. Hepatology 48: 2047-2063, 2008.

4. Hauptman N and Glavac D: MicroRNAs and long non-coding RNAs: prospects in diagnostics and therapy of cancer. Radiol Oncol 47: 311-318, 2013.

5. Wang W, Lin H, Zhou L, et al: MicroRNA-30a-3p inhibits tumor proliferation, invasiveness and metastasis and is downregulated in hepatocellular carcinoma. Eur J Surg Oncol: Nov 19, 2013 (Epub ahead of print).

6. Ke Y, Zhao W, Xiong J and Cao R: Downregulation of miR-16 promotes growth and motility by targeting HDGF in non-small cell lung cancer cells. FEBS Lett 587: 3153-3157, 2013.

7. Yang H, Li Q, Zhao W, Yuan D, Zhao H and Zhou Y: miR-329 suppresses the growth and motility of neuroblastoma by targeting KDM1A. FEBS Lett 588: 192-197, 2014.

8. Guo Y, Ying L, Tian Y, et al: miR-144 downregulation increases bladder cancer cell proliferation by targeting EZH2 and regulating Wnt signaling. FEBS J 280: 4531-4538, 2013.

9. Iwaya T, Yokobori T, Nishida N, et al: Downregulation of miR-144 is associated with colorectal cancer progression via activation of mTOR signaling pathway. Carcinogenesis 33: 2391-2397, 2012.

10. Zha W, Cao L, Shen Y and Huang M: Roles of Mir-144-ZFX pathway in growth regulation of non-small-cell lung cancer. PLoS One 8: e74175, 2013.

11. Katayama Y, Maeda M, Miyaguchi K, et al: Identification of pathogenesis-related microRNAs in hepatocellular carcinoma by expression profiling. Oncol Lett 4: 817-823, 2012.

12. Zhang LY, Ho-Fun Lee V, Wong AM, et al: MicroRNA-144 promotes cell proliferation, migration and invasion in nasopharyngeal carcinoma through repression of PTEN. Carcinogenesis 34: 454-463, 2013.

13. Hers I, Vincent EE and Tavaré JM: Akt signalling in health and disease. Cell Signal 23: 1515-1527, 2011.

14. Stambolic V and Woodgett JR: Functional distinctions of protein kinase $\mathrm{B} / \mathrm{Akt}$ isoforms defined by their influence on cell migration. Trends Cell Biol 16: 461-466, 2006.

15. Nassirpour R, Mehta PP and Yin MJ: miR-122 regulates tumorigenesis in hepatocellular carcinoma by targeting AKT3. PLoS One 8: e79655, 2013 